\title{
Prevalence and factors predictive of intraocular fungal infection in patients with fungemia at an academic urban tertiary care center
}

This article was published in the following Dove Press journal:

Clinical Ophthalmology

29 September 2015

Number of times this article has been viewed

\author{
Elena Geraymovych' \\ Joseph H Conduff ${ }^{2}$ \\ Puneet S Braich ${ }^{3}$ \\ Christopher T Leffler ${ }^{3}$ \\ Vikram S Brar ${ }^{3}$ \\ 'Department of Ophthalmology, \\ University of Texas Health Science \\ Center at San Antonio, San Antonio, \\ TX, ${ }^{2}$ Virginia Commonwealth \\ University School of Medicine, \\ ${ }^{3}$ Department of Ophthalmology, \\ Virginia Commonwealth University, \\ Richmond, VA, USA
}

Objective: To report the prevalence and to identify factors predictive of intraocular infection in patients with fungemia receiving prophylactic antifungal therapy.

Methods: A retrospective review of patients who received prophylactic antifungal therapy and a dilated fundus examination at an academic urban tertiary care center from 2000 to 2007. Basic demographic information, fungal species grown, antifungal agent(s) used, number of positive blood culture specimens, visual acuity, visual symptoms, and known risks of disseminated candidiasis were noted. Logistic regression analysis was used to determine the factors significantly associated with intraocular fungal infection.

Results: A total of 132 patients with positive fungemia culture were requested to have ophthalmology consults. The prevalence of ocular infection was $6.9 \%(\mathrm{~N}=9)$. All nine patients were infected with Candida species. Undergoing gastrointestinal (GI) surgery within the prior 6 months was significantly related to developing intraocular infection, with an odds ratio of 18.5 ( $95 \%$ confidence interval, 15.1-24.3; $P=0.002)$. Having $\geq 3$ positive fungal blood cultures was also a significant risk factor, with an odds ratio of 2.6 (95\% confidence interval, $1.8-3.7 ; P=0.03)$. Among 40 patients having GI surgery, eight $(20.0 \%)$ had intraocular fungal disease, compared with one of 92 patients (1.1\%) not having GI surgery. Among 125 patients with a negative baseline examination result, two of 32 patients $(6.3 \%)$, who had recent GI surgery, subsequently developed fungal ocular disease, compared with 0 of 93 patients $(0 \%)$, who did not have recent GI surgery.

Conclusion: Recent GI surgery and higher numbers of positive fungal blood culture specimens may be predictive of candida ocular infections. Normal baseline fundoscopy examination results in patients with such risks may require repeat evaluations to detect delayed manifestations.

Keywords: fungal chorioretinitis, fungal endophthalmitis, risks factors for intraocular fungal infection, risks of gasterointestinal surgery

\section{Introduction}

One of the most common inpatient ophthalmology consults is for the fundoscopic evaluation of patients with fungemia. ${ }^{1,2}$ Candida species, in particular, are the fourth most common organisms identified on microbial blood stream infections in temperate climates of the USA and Europe. ${ }^{3-5}$ Some of the major risk factors for the development of candidemia are immunosuppression, indwelling catheters, total parenteral nutrition, and intravenous drug abuse. ${ }^{6-8}$ Ocular infection from fungemia is uncommon, but if present, permanent vision loss may result depending on the location and extent. The current recommendation from the Infectious Disease Society of America (IDSA) is to provide at least one dilated fundus examination to patients with positive fungal blood cultures. ${ }^{9}$ Chorioretinitis and endophthalmitis are the most common manifestations of fungemia in the eye. ${ }^{2,3,6}$ These entities were more prevalent before the widespread
Correspondence: Vikram S Brar

Department of Ophthalmology, Virginia

Commonwealth University, 40 I N

II th Street, Suite 439, Richmond,

VA 23298, USA

Tel + I 8048289315

Fax + I 8048281010

Email vbrar@mcvh-vcu.edu 
use of prophylactic antifungal therapy, quicker laboratory detection of fungemia, and newer generation agents with greater efficacy and better side-effect profiles. ${ }^{10-12}$

A positive ophthalmic examination result in patients with fungemia, in addition to facilitating management of secondary ocular complications, establishes the diagnosis of disseminated candidiasis. ${ }^{1}$ However, the utility of routine ophthalmology consultation to rule out ocular involvement in patients with fungemia has been challenged by many authors..$^{8,11,13-15}$ Although formal cost-effectiveness analyses on this issue are lacking, there is an increasing sentiment expressed in the ophthalmic literature to change the criteria by which an ophthalmology evaluation is requested for inpatients with fungemia. ${ }^{13,15}$

The reliance of visual symptoms alone results in a poor sensitivity $(28.6 \%)$ and poor positive predictive value $(26.7 \%)$ as the majority of patients with ocular infection may have no symptoms or external signs. ${ }^{13}$ The problem of relying on symptoms and external signs is compounded when patients are preverbal or unresponsive due to intubation or other medical comorbidities. ${ }^{8-10}$

With respect to patients with fungemia and specifically candidemia, there may be a subset of risk factors that increases the likelihood of detecting intraocular infection. Since the prevalence of disease affects pretest likelihood, a determination of the local prevalence of endogenous ocular fungal infections would be necessary to guide any changes to the screening algorithm of patients with fungemia. ${ }^{16}$

Our aims for this study were twofold: 1) to report the prevalence of endogenous fungal chorioretinitis/endophthalmitis among patients with fungemia and 2) to determine what risk factors among patients with fungemia place them at greater odds of having intraocular infection.

\section{Methods}

This study was a retrospective review conducted at the Virginia Commonwealth University (VCU) Medical Center between 2000 and 2007 that enrolled all inpatients consulted by the ophthalmology department to rule out ocular fungal infections. Consult sheets, electronic records, laboratory results, discharge summaries, and billing statements were reviewed. Patients with any prior ocular surgery, prior ocular trauma, and negative fungemia culture results, despite external candidal infections (ie, oral or urine infections), were excluded from participation. This study was approved by the VCU Office of Research Subjects Protection and was in accordance with the Declaration of Helsinki.

All abnormal fundoscopic examinations were confirmed by a faculty member who had undergone training in a vitreoretinal fellowship. Visual acuity was assessed with standard near cards at the bed side or Snellen charts in the clinic. In preverbal children, it was recorded whether or not they could fix and follow. When patients were intubated, sedated, or both, vision was recorded as unable to assess. An anterior segment examination was done with an external light source (eg, indirect ophthalmoscope with 20D lens), portable slit lamp, or mounted slit lamp in clinic. All dilated fundus examinations were performed with indirect ophthalmoscopy. The diagnosis of fungal chorioretinitis was based on the description of Donahue et $\mathrm{al}^{11}$ as a deep focal, fluffy white lesion in the chorioretinal layers. Endophthalmitis was defined as vitreous involvement and further anterior extension with fluffy vitreous balls, vitreous haze, vitreous abscess, anterior chamber cells, or hypopyon. Basic demographic information was gathered along with fungal species grown, antifungal agent(s) used, number of positive blood culture specimens, visual acuity, and visual symptoms. Further, we documented historically reported risk factors (HRRF) $)^{6-8}$ for candidemia such as broad spectrum antibiotic use, gastrointestinal (GI) surgery within the previous 6 months, generalized immunosuppression, intravenous drug abuse, indwelling intravenous catheters, parenteral hyperalimentation, and multiple-organ involvement.

For the purposes of this study, GI surgery consisted of incisional surgery anywhere from the oral cavity to the anus as well as endoscopic procedures of this area. Etiologies of immunosuppression consisted of being on chronic corticosteroid therapy, routine use of immunosuppressive agents in patients posttransplant or with autoimmune conditions, chemotherapeutic agents, and infection with HIV. Further, diabetes mellitus was also considered as a form of immune suppression. VCU Medical Center follows the recommendations of the IDSA and the Surgical Infection Society, ${ }^{17}$ regarding acquisition of specimens for potential blood stream infections, obtaining 20-30 mL of blood with 2-3 bottles. If multiple sites were used to gather samples, they were all accessed within 1 hour of each other. If one of the samples was obtained from a venous catheter, then a separate bottle was also needed from a venipuncture site as the risk of contamination is higher for indwelling venous lines. Patients older than 2 months of age had the venipuncture site disinfected with chlorhexidine or $2 \%$ iodine tincture. Changes in the clinical picture of the patient may have resulted in repeat blood culture sampling, which is how some patients in this study could have had more than three bottles of blood sampled.

Besides determining the prevalence of ocular fungal infection in our population, we performed a logistic 
regression analysis to determine the possible risk factors that would impact the odds of having ocular involvement. The dependent variable was the presence of intraocular fungal infection (fungal chorioretinitis/endophthalmitis), and the independent variables were the demographic factors (Table 1) and the HRRF for disseminated candidemia. ${ }^{6-8}$ One variable was added to the model, besides the above, the proportion of blood culture positive specimens obtained. With these

Table I Patient characteristics

\begin{tabular}{|c|c|c|c|}
\hline & $N=123(\%)$ & $\mathbf{N}=9$ (\%) & $P$-value \\
\hline \multicolumn{4}{|l|}{ Sex } \\
\hline Men & $72(58.5)$ & $4(44.4)$ & 0.42 \\
\hline Women & $52(4 \mid .5)$ & $5(55.6)$ & 0.42 \\
\hline Age, \pm standard deviation (years) & $37.3 \pm 25.2$ & $51.1 \pm 17.8$ & 0.05 \\
\hline $\begin{array}{l}\text { Age after exclude those } \leq 20 \text { years old, none of } \\
\text { whom had ocular fungal infection }\end{array}$ & $45.6 \pm 19.8$ & $51.1 \pm 17.8$ & 0.39 \\
\hline \multicolumn{4}{|l|}{ Race } \\
\hline White & $49(39.8)$ & $4(44.4)$ & 0.75 \\
\hline Black & $64(52.0)$ & $4(44.4)$ & 0.66 \\
\hline Other & $10(8.1)$ & $I(I I . I)$ & 0.79 \\
\hline Visual acuity (near, best corrected) & $N=$ number of eyes; $247(\%)$ & $\mathbf{N}=$ number of eyes; $17(\%)$ & \\
\hline $20 / 20-20 / 40$ & $104(42.1)$ & $8(47.1)$ & 0.69 \\
\hline $20 / 50-20 / 200$ & $38(15.4)$ & $4(23.5)$ & 0.38 \\
\hline$>20 / 200$ & $4(1.6)$ & 0 & 0.60 \\
\hline Fixes and follows & $24(9.7)$ & 0 & 0.18 \\
\hline Unable to assess & $77(3 \mid .2)$ & $6(35.3)$ & 0.72 \\
\hline \multicolumn{4}{|c|}{ Visual symptoms (floaters, photopsias, pain, and conjunctival injection) } \\
\hline Present & $76(30.8)$ & $4(23.5)$ & 0.74 \\
\hline Absent & $94(38.1)$ & $10(58.5)$ & 0.09 \\
\hline Unable to assess & $77(31.2)$ & $3(17.6)$ & 0.24 \\
\hline Candida species grown & $\mathrm{N}=123(\%)$ & $\mathbf{N}=\mathbf{9}(\%)$ & \\
\hline C. Albicans & $50(40.7)$ & $6(66.7)$ & 0.13 \\
\hline C. Parapsilosis & $30(24.4)$ & 0 & 0.09 \\
\hline C. Glabrata & $22(17.9)$ & $2(22.2)$ & 0.75 \\
\hline C. Tropicalis & $15(12.2)$ & $I(I I . I)$ & 0.92 \\
\hline C. Lusitaniae & $2(1.6)$ & 0 & 0.65 \\
\hline C. Krusei & $2(1.6)$ & 0 & 0.65 \\
\hline Candida not otherwise specified & $2(1.6)$ & 0 & 0.65 \\
\hline \multicolumn{4}{|l|}{ Prophylactic antifungal therapy used ${ }^{a}$} \\
\hline Amphotericin B & $30(24.4)$ & $I(I I . I)$ & 0.36 \\
\hline Caspofungin & $48(39.0)$ & $4(44.4)$ & 0.75 \\
\hline Fluconazole & $61(49.6)$ & $5(55.5)$ & 0.73 \\
\hline Voriconazole & $13(10.6)$ & $I(I I . I)$ & 0.96 \\
\hline Number using multiple antifungal agents & $36(29.2)$ & $2(22.2)$ & 0.65 \\
\hline \multicolumn{4}{|l|}{ Risk factors for disseminated candidiasis ${ }^{\mathrm{a}}$} \\
\hline Broad spectrum antibiotic use & $98(79.7)$ & $8(77.8)$ & 0.89 \\
\hline Indwelling intravenous catheters & $92(74.7)$ & $7(66.7)$ & 0.59 \\
\hline Gastrointestinal surgery within the last & $32(26.0)$ & $8(88.9)$ & $<0.01$ \\
\hline \multicolumn{4}{|l|}{6 months } \\
\hline Parenteral hyperalimentation & $59(47.9)$ & $5(55.5)$ & 0.33 \\
\hline Generalized immunosuppression & $63(51.2)$ & $6(66.7)$ & 0.10 \\
\hline Multiple-organ involvement & $40(32.5)$ & $4(44.4)$ & 0.16 \\
\hline Intravenous drug abuse & $2(0.02)$ & 0 & 0.60 \\
\hline $\begin{array}{l}\text { Average number of positive blood culture } \\
\text { specimens per patient }\end{array}$ & 2.02 & 4.22 & 0.03 \\
\hline Fraction of positive blood culture specimens & 0.52 & 0.78 & 0.04 \\
\hline Intraocular pressure & $14.0 \mid$ & 13.20 & 0.71 \\
\hline Concomitant bacteremia & $28(22.6)$ & $I(I I . I)$ & 0.34 \\
\hline Number of nonocular surgeries in the past year & 0.86 & 1.55 & 0.07 \\
\hline
\end{tabular}

Notes: Columns with mutual exclusively may not round to $100 \%$ due to rounding errors. ${ }^{\text {TT }}$ The following columns will not add to $100 \%$ since these items were not mutually exclusive. 
parameters defined, a backward selection technique was used to select the most parsimonious model as nonsignificant covariates were excluded. We also used multiple two-sided independent $t$-tests to compare patients with ocular infection to those without. A significance of $P<0.05$ was used. All statistical analyses were done with SAS 9.3 (2011 SAS Institute Inc., Cary, NC, USA).

\section{Results}

A total of 132 patients with fungemia during the study period had an inpatient evaluation by the ophthalmology department. Nine of 132 patients $(6.9 \%)$ had intraocular fungal infection. Five of these nine were female patients. Four of the nine were Black, four were White, and one was categorized as "Other" (Table 1). The mean age of patients with ocular infection was 51.1 years with a standard deviation of 16.8 . Seven patients had bilateral chorioretinitis, one patient had chorioretinitis of the left eye only, and another had bilateral fungal endophthalmitis. All nine cases were infected with Candida species and Candida albicans grew in six patients. Caspofungin was used in four of the nine patients, and fluconazole was used in five. The patients on caspofungin were either already on fluconazole or later changed to fluconazole, upon the diagnosis of ocular candidiasis. The patient with endophthalmitis died 4 weeks after detection, and a patient with bilateral chorioretinitis died 8 weeks after detection. Four patients resolved with antifungal therapy alone, and three patients were lost to follow-up. None of the patients received intravitreal antifungal therapy or pars plana vitrectomy. Two of nine patients had normal baseline examination results and went on to develop positive fundoscopic findings for candidal involvement within 14 days. Both had recent GI surgery. In one of these two patients, a repeat examination was requested 11 days later for bilateral photopsias, which ended up yielding evidence of bilateral chorioretinitis. The other patient remained asymptomatic, and positive examination findings were found on routine outpatient examination 14 days after baseline examination. Only one other patient with ocular fungal disease was symptomatic, also complaining of bilateral photopsias but had a positive baseline examination.

A significantly greater proportion of patients with endogenous candidal ocular infection had recent GI surgery compared to those without ocular infection ( $88.9 \%$ vs $26.0 \%$, $P<0.01)$. Compared to patients without fungal eye disease, the mean fraction of positive blood culture specimens was significantly higher among patients with fungal eye disease ( 0.78 vs $0.52, P=0.04)$. Some differences between those with ocular infection compared to those without ocular involvement approached significant levels, such as the number of nonocular surgeries ( 1.55 vs $0.86, P=0.07)$ and generalized immune suppression (66.7\% vs $51.2 \%, P=0.10)$. C. albicans represented $40.7 \%$ of the species among patient without intraocular infection and $66.7 \%$ of patients with intraocular infection. However, the type of Candida species did not impact the risk of developing ocular infection (Tables 1 and 2). Fluconazole was used in 66 of 132 of our patients, yet the type of antifungal agent used in patients with fungemia did not significantly impact the development of intraocular fungal infection. In the logistic regression analysis, only three factors significantly affected the fit of the model regarding the presence of ocular fungal infection (Table 2). Patients who had undergone GI surgery within the last 6 months were at an 18.5 times higher odds of developing ocular fungal infection compared to those who did not have GI surgery in the last 6 months $(P=0.002)$. Patients who had grown $\geq 3$ positive blood culture specimens were at a 2.6 times higher odds of developing ocular fungal infection compared to those with $\leq 2$ positive specimens $(P=0.03)$. The fraction of positive blood culture specimens was also associated with the presence of ocular fungal infection. When three-quarters or more of the blood cultures were positive, the odds of having ocular candidal infection were more than twice as high $(P=0.04)$. None of the remaining HRRF for candidiasis had an impact on intraocular candidal infection.

Table 2 Factors impacting ocular Candida infection in the multivariate logistic regression model

\begin{tabular}{lll}
\hline & Odds ratio (95\% confidence intervals) & P-value \\
\hline Gastrointestinal surgery & & - \\
$\quad$ None in prior 6 months (reference) & - & 0.002 \\
$\quad$ One or more in the prior 6 months & & \\
Number of positive Candida blood cultures & - & - \\
$\quad \leq 2$ (reference) & $2.6(I .8-3.7)$ & 0.03 \\
$\geq 3$ & - & - \\
Fraction of positive blood culture specimens & - & 0.04 \\
$\quad<0.75$ (reference) & $2.4(1.2-3.1)$ & \\
$\geq 0.75$ &
\end{tabular}

Notes: Odds ratios and $95 \%$ confidence intervals for the covariates associated with an increased risk of intraocular fungal infection. $P$-values are from the $\chi^{2}$ tests. 


\section{Discussion}

In accordance with the recommendations of the IDSA, all patients with fungemia at our institution receive at least one dilated eye examination. ${ }^{9}$ Moreover, some reports state that up to $18 \%$ of ocular candidal infections have negative baseline fundoscopy examinations. ${ }^{18}$ The time from a negative fundoscopy examination to visible ocular fungal lesions has ranged from 8 days to several weeks. ${ }^{19-21}$ Given the chance of delayed presentation, we repeat a dilated fundus examination at 4-6 weeks if the baseline fundoscopy examination is negative.

The prevalence of ocular fungal infection in our population was $6.9 \%$. All cases were from Candida species. Although this figure is higher than some recent studies, this prevalence seems to be in line with several contemporary reports that demonstrate values from $2 \%$ to $16 \%$. ${ }^{1,2,8,12,15,22,23}$ The prevalence of current reports is lower than historical reports $(35 \%-45 \%) .{ }^{19,24-26}$ This is largely due to the initiation of prophylactic antifungal therapy for all positive blood cultures. . 15,19,22-26 $^{2}$

Many authorities are questioning the utility of ophthalmic examination for patients with fungemia since now there is quicker laboratory detection of fungal blood specimens prompting the use of antifungal agents, which have greater efficacy and better side-effect profiles compared to decades ago.,11,13-15 Furthermore, some authors assert that patients with endogenous spread of fungal disease to the eye are at higher risk of mortality, and an ocular examination may add to the cost of end of life care. Oude Lashof et $\mathrm{al}^{12}$ reported the 14-week mortality of patients with endogenous candidal endophthalmitis to be $50 \%$, while that in ocular candidiasis, which was not classified as endophthalmitis, was $43.3 \%$. The latter values were not significantly higher than the 14-week mortality rate for patients with candidemia but without ocular infection, $36.5 \%(P=0.31)$.

Moreover, because of these advances along with the higher mortality among patients with fungemia, the management is reported to rarely change even after fundoscopic evidence of ocular infection. Ghodasra et $\mathrm{al}^{13}$ estimated that an average of 26.4 patients with fungemia would need to be evaluated, at an average cost of 5,620.33 US Dollars per patient, in order to find one needing a change in management. This money value was based on the Centers of Medicare and Medicaid Services 2014 Physician Fee Schedule. ${ }^{27}$ These authors, among many others, are reassessing the utility of fundoscopic examinations for patients with fungemia.

However, given the potentially negative visual outcome of intraocular fungal infections, eliminating the need for fundoscopic examinations altogether may be premature. Perhaps, there are a unique set of risk factors that would raise the pretest likelihood, or better stated, the pre-fundoscopy examination likelihood of a patient with candidemia to have ocular involvement.
Our study demonstrated that of the HRRF, only recent GI incisional or endoscopic surgery was significantly associated with ocular candidal infection (odds ratio, 18.5; 95\% confidence interval, $15.1-24.3 ; P=0.002)$. It is possible that Candida species that are commensal with the GI tract could gain access to the blood stream in high enough inoculations to cause systemic disease.

A higher number of positive candidal blood culture specimen bottles also strongly correlated with the presence of intraocular infection. Having $\geq 3$ positive culture bottles increased the odds of ocular infection compared to those with $\leq 2$ positive culture bottles. Greater numbers of positive culture specimens may be a marker for larger fungal inoculation, or in the circumstance of delayed specimen collection by a few days, it could represent a virulent organism and/or an immunocompromised host. This may predispose the patient to disseminated fungal disease. The fraction of positive culture bottles can also be understood by the above statements.

There are several noteworthy limitations to our study. First, only patients suspected of having abnormal fundus examinations based on an evaluation by a resident ophthalmologist were subjected to a confirmatory examination by a vitreoretinal specialist. It is possible that patients deemed as having normal fundus examinations may have had subtle retinal lesions that a novice examiner may have overlooked. Although our policy was to have outpatient follow-up for inpatients not seen by an attending physician, not all patients were able to make such appointments.

Second, many patients with fungemia, especially those with disseminated disease, may be in critical condition. It is possible that we did not evaluate all patients with fungemia as they may have had significant comorbidities that took precedence over ophthalmic consultation or were deceased before such consultation could take place. These patients by virtue of their aggressive disease could have been significantly different from the patients who had been evaluated by the ophthalmology service. Finally, the nature of retrospective investigations lends itself to inconsistencies in documentation, which leads to suboptimal data entry for analysis.

In conclusion, this study demonstrated the prevalence of intraocular infection to be $6.9 \%$ among patients with fungemia in our urban academic medical center. Many clinicians are reevaluating the utility of inpatient ophthalmology consults on patients with fungemia. However, we feel that missing positive cases increases the risk of morbidity to the eye, especially in cases of asymptomatic endophthalmitis, and mortality to the patient ${ }^{13}$ by missing the chance to establish a diagnosis of disseminated candidiasis. The presence of certain risk factors may raise the likelihood of a patient with fungemia, and candidemia 
in particular, of having ocular infection. This study showed that patients having GI surgery within the last 6 months, having $\geq 3$ positive candida blood culture specimens, and having positive Candida blood cultures in $\geq 0.75$ of specimens collected, may all increase the risk of ocular candidal infection, and thereby warrant greater scrutiny. When patients with such risk factors have negative baseline fundoscopic examinations, ophthalmologists may wish to perform repeat examinations within 2 weeks to detect delayed onset disease as is the current practice at our institution. For patients without such risks, the need for repeat fundoscopic examinations may continue to be at the discretion of the clinician on a case-by-case basis. Lastly, prospective research is necessary to determine which other risk factors are significantly associated with ocular infection, thus may be identified as predictive factors and may better guide clinical decision-making when reconsidering screening algorithms for patients with fungemia.

\section{Disclosure}

The authors report no conflicts of interest in this work.

\section{References}

1. Carter K, Miller K. Ophthalmology inpatient consultation. Ophthalmology. 2001;108:1505-1511.

2. Grewal DS, Chiang E, Wong E, et al. Adult ophthalmology inpatient consults at a tertiary care teaching hospital. Ophthalmology. 2014;121: 1489-1491.

3. Gumbo T, Isada CM, Hall G, Karafa MT, Gordon SM. Candida glabrata Fungemia. Clinical features of 139 patients. Medicine (Baltimore). 1999; 78(4):220-227.

4. Pfaller MA, Jones RN, Doern GV, et al. International surveillance of blood stream infections due to Candida species in the European SENTRY Program: species distribution and antifungal susceptibility including the investigational triazole and echinocandin agents. SENTRY Participant Group (Europe). Diagn Microbiol Infect Dis. 1999;35(1):19-25.

5. Zirkel J, Klinker H, Kuhn A, et al. Epidemiology of Candida blood stream infections in patients with hematological malignancies or solid tumors. Med Mycol. 2012;50(1):50-55. doi: 10.3109/13693786.2011.587211.

6. Hariprasad SM, Mieler WF, Holz ER, et al. Determination of vitreous, aqueous, and plasma concentration of orally administered voriconazole in humans. Arch Ophthalmol. 2004;122(1):42-47.

7. Brod RD, Clarkson JG, Flynn HW, Miller D. Endogenous fungal endophthalmitis. In: Tasman W, editor. Duane's Clinical Ophhthalmology. Philadelphia, PA: JB Lippincott Co; 1994:14-16.

8. Feman SS, Nichols JC, Chung SM, Theobald TA. Endophthalmitis in patients with disseminated fungal disease. Trans Am Ophthalmol Soc. 2002;100:67-70.

Clinical Ophthalmology

\section{Publish your work in this journal}

Clinical Ophthalmology is an international, peer-reviewed journal covering all subspecialties within ophthalmology. Key topics include: Optometry; Visual science; Pharmacology and drug therapy in eye diseases; Basic Sciences; Primary and Secondary eye care; Patient Safety and Quality of Care Improvements. This journal is indexed on Submit your manuscript here: http://www.dovepress.com/clinical-ophthalmology-journal
9. Pappas PG, Kauffman CA, Andes D, et al; Infectious Diseases Society of America. Clinical practice guidelines for the management of candidiasis: 2009 update by the Infectious Diseases Society of America. Clin Infect Dis. 2009;48:503-535.

10. Dozier CC, Tarantola RM, Jiramongkolchai K, Donahue SP. Fungal eye disease at a tertiary care center: the utility of routine inpatient consultation. Ophthalmology. 2011;118:1671-1676.

11. Donahue SP, Greven CM, Zuravleff JJ, et al. Intraocular candidiasis in patients with candidemia: clinical implications derived from a prospective multicenter study. Ophthalmology. 1994;101:1302-1309.

12. Oude Lashof AM, Rothova A, Sobel JD, et al. Ocular manifestations of candidemia. Clin Infect Dis. 2011;53:262-268.

13. Ghodasra DH, Eftekhari K, Shah AR, VanderBeek BL. Outcomes, impact on management, and costs of fungal eye disease consults in a tertiary care setting. Ophthalmology. 2014;121(12):2334-2339.

14. Scherer W, Lee K. Implications of early systemic therapy on the incidence of endogenous fungal endophthalmitis. Ophthalmology. 1997; 104:1593-1598.

15. Huynh N, Chang H, Borboli-Gerogiannis S. Ocular involvement in hospitalized patients with candidemia: analysis at a Boston tertiary care center. Ocul Immunol Inflamm. 2012;20:100-103.

16. Westover MB, Eiseman NA, Cash SS, Bianchi MT. Information theoretic quantification of diagnostic uncertainty. Open Med Inform J. 2012;6:36-50.

17. Solomkin JS, Mazuski JE, Bradley JS, et al. Diagnosis and management of complicated intra-abdominal infection in adults and children: guidelines by the Surgical Infection Society and the Infectious Diseases Society of America. Surg Infect (Larchmt). 2001;11(1):79-109.

18. Braich PS, Chang JS, Albini TA, Schefler AC. Irido-lenticular abscess as the initial sign of Candida albicans endogenous endophthalmitis. Ophthalmic Surg Lasers Imaging. 2011;42 Online:e107-9.

19. Parke DW, Jones DB, Gentry LO. Endogenous endophthalmitis among patients with candidemia. Ophthalmology. 1982;89:789-796.

20. Brooks RG. Prospective study of Candida endophthalmitis in hospitalized patients with candidemia. Arch Intern Med. 1989;149:2226-2228.

21. Krishna R, Amuh D, Lowder CY, Gordon SM, Adal KA, Hall G. Should all patients with candidaemia have an ophthalmic examination to rule out ocular candidiasis? Eye (Lond). 2000;14:30-34.

22. Donahue SP, Hein E, Sinatra RB. Ocular involvement in children with candidemia. Am J Ophthalmol. 2003;135:886-887.

23. Rodríguez-Adrián LJ, King RT, Tamayo-Derat LG, Miller JW, Garcia CA, Rex JH. Retinal lesions as clues to disseminated bacterial and Candida infections: frequency, natural history, and etiology. Medicine (Baltimore). 2003;82:187-202.

24. Klein J, Watanakunakorn C. Hospital-acquired fungemia: its natural course and clinical significance. Am J Med. 1979;67:51-58.

25. Henderson D, Edwards J, Montomerie J. Hematogenous Candida endophthalmitis in patients receiving parenteral hyperalimentation fluids. J Infect Dis. 1981;143:655-661.

26. McDonnell P, McDonnell J, Brown R, Green W. Ocular involvement in patients with fungal infections. Ophthalmology. 1985;92:706-709.

27. Centers for Medicare and Medicaid Services. Physician Fee Schedule Search [database online]; 2008. Available from: https://www.cms. gov/apps/physician-fee-schedule/search/search-criteria.aspx. Accessed December 18, 2014.
PubMed Central and CAS, and is the official journal of The Society of Clinical Ophthalmology (SCO). The manuscript management system is completely online and includes a very quick and fair peer-review system, which is all easy to use. Visit http://www.dovepress.com/ testimonials.php to read real quotes from published authors. 